\title{
Epidemiological and Clinical Characteristics of Deceased COVID-19 Patients
}

\author{
Mohammed Badedi (iD) \\ Hussain Darraj $\mathbb{D}^{\prime}$ \\ Awaji Qasem Alnami ${ }^{1}$ \\ Ali Makrami' \\ Mohamed Salih Mahfouz $\mathbb{D}^{2}$ \\ Khalid Alhazmi ${ }^{2}$ \\ Nahid Mahmoud' \\ Halimh Mosa' \\ 'General Directorate of Health Affairs, \\ Jazan, Jazan Region, Saudi Arabia; ${ }^{2} J a z a n$ \\ University, Jazan, Jazan Region, Saudi \\ Arabia
}

\begin{abstract}
Objective: Fatalities due to coronavirus disease 2019 (COVID-19) continue to increase, and information on the epidemiological and clinical characteristics of deceased patients who were hospitalized with COVID-19 is limited in the Arab region. The current study aimed to address this gap.

Methods: Three hundred and four Saudi patients in Jazan Region, Saudi Arabia, who died after being hospitalized with COVID-19 between July 1, 2020, and December 31, 2020, were analyzed in this retrospective cohort study.

Results: A greater proportion of male patients (59\%), compared to female patients (41\%), died due to COVID-19. Just over half (55\%) of the deaths due to COVID-19 affected patients aged $\geq 65$ years. More than two-thirds of the deceased COVID-19 patients had diabetes $(70 \%)$ and hypertension (69\%); other comorbidities were obesity $(30 \%)$, heart disease (30\%), and chronic kidney disease (14\%). Dyspnea (91\%), cough (80\%), and fever $(70 \%)$ were the most frequently reported clinical symptoms. Eighty-five per cent of COVID19 deaths occurred in patients admitted to the intensive care unit (ICU), and $90 \%$ of the patients required mechanical ventilation. Typically, lymphopenia, and neutrophilia were observed on admission and 24 hours prior to death. Creatinine and serum ferritin levels and erythrocyte sedimentation rate and D-dimer plasma levels increased significantly following infection with COVID-19. Lung infiltrates and pulmonary opacity (83\%) were the most common findings on chest X-ray. Respiratory failure $(70 \%)$ and acute respiratory distress syndrome (52\%) were the leading complications to death. Logistic and Cox regression revealed that a higher age, smoking, high creatinine and aspartate transaminase levels, and respiratory failure were significantly associated with the risk of mortality during the early stay in hospitals.
\end{abstract}

Conclusion: The proportion of comorbidities was high in deceased patients who were hospitalized with COVID-19 in Jazan region, Saudi Arabia. A higher age, smoking, and respiratory failure were significant predictors of mortality during the early stay in hospitals. Keywords: COVID-19, SARS-CoV-2, deaths, mortality

\section{Introduction}

Coronavirus disease 2019 (COVID-19), a novel infectious disease caused by severe acute respiratory syndrome coronavirus 2 (SARS-CoV-2), ${ }^{1}$ has become a global public health concern as the number of deaths due to COVID-19 continues to increase. In February 2021, the COVID-19 mortality rate was estimated to be roughly $3 \%$ globally. ${ }^{1}$ By February 2021, the Saudi Ministry of Health reported over 6000 confirmed COVID-19 deaths in Saudi Arabia and, of these, 475 occurred in Jazan Region, representing a $4 \%$ mortality rate. ${ }^{2}$
Correspondence: Mohammed Badedi Email dr.badedi@gmail.com 
The clinical features and severity of COVID-19 vary among individuals, based on multiple factors, such as age and associated comorbidities. ${ }^{3}$ Asymptomatic COVID-19 patients may not require hospitalization. ${ }^{4-6}$ However, severe cases, for example, patients with systemic inflammation, pneumonia, hypoxemia, and peripheral oxygen saturation $(\mathrm{SpO} 2)$ of $<92 \%$, require hospitalization. ${ }^{7}$ Patients with severe COVID-19 may become critically ill with acute respiratory distress, shock, myocardial injury, heart failure, coagulation dysfunction, and acute kidney injury, all of which may lead to death. ${ }^{7}$

Knowledge of the epidemiological and clinical features of patients who demise due to COVID-19 is needed for a better understanding and proper management of the disease, and these features have been evaluated in a few studies. ${ }^{8,9}$ However, there is a need to update and increase the limited available evidence on the epidemiological and clinical features of patients who die due to COVID-19, particularly in Saudi Arabia, the largest country in the Arabic peninsula, owing to genetic and ethnic differences among populations, the paucity of data in this regard, and the rate at which the virus is mutating and evolving. In addition, to the best of our knowledge, the epidemiological and clinical characteristics of Saudi patients who have died due to COVID-19 in Jazan Region, Saudi Arabia, has not been evaluated in any prior study. Thus, the present study aimed to address epidemiological and clinical characteristics of deceased COVID-19 patients in Jazan region, Saudi Arabia, and to identify mortality predictors during the early stay in hospitals.

\section{Method}

All Saudi patients confirmed to have died due to COVID-19 (n =304), in Jazan Region, Saudi Arabia, between July 1, 2020, and December 31, 2020, were included from fourteen secondary care hospitals in this retrospective cohort study that sought to determine their epidemiological and clinical characteristics. Jazan Region is situated in southwestern Saudi Arabia and is characterized by a relatively homogenous population with similar ethnic and socioeconomic characteristics. ${ }^{10-12}$ In the current study, a diagnosis of COVID-19 was confirmed by the identification of viral RNA in a nasopharyngeal swab sample using real-time reverse transcription-polymerase chain reaction (LightCycler ${ }^{\circledR} 480$ Instrument II, Roche).

The data were collected retrospectively from medical records and included demographic data, age, sex, smoking history, blood group, comorbidities, clinical symptoms, laboratory test results, radiological findings, treatment medications, and period of hospitalization. Coorbidities included diabetes, hypertension, cardiovascular disease, chronic kidney disease, cancer, and obesity. The most prevalent clinical COVID-19 symptoms were fever, cough, dyspnea, chest pain, diarrhea, and confusion. The laboratory tests evaluated complete blood count, coagulation profile, and renal and liver function. The dates of COVID-19 diagnosis, hospital admission, and death were also recorded. The survival time during hospitalization (length of stay) was defined as the period between the date of admission and death. It was categorized by median into death during short stay and long stay hospitalization, this was similar to a systemic review study that pooled many studies across the world. ${ }^{13}$ In keeping with the World Health Organization's guidelines, ${ }^{14}$ body mass index (BMI) was calculated as weight in kilograms divided by the square of the person's height in meters. A BMI score of $\geq 30 \mathrm{~kg} / \mathrm{m} 2$ was categorized as obese. The Jazan Health Ethics Committee (Reference number: H-10-Z-073) granted ethical approval (No. 2030) for the study to be conducted, and the research complied with the Declaration of Helsinki. Consent was waived as data were anonymous and collected retrospectively (secondary data).

Data entry and analysis were performed using Statistical Package for the Social Sciences ${ }^{\circledR}$ software. ${ }^{15}$ The categorical variables were presented as percentages, and the continuous variables were described using mean \pm standard deviation (SD) for the normally distributed variables and the median (interquartile range $[\mathrm{IQR}]$ ) for the non-normally distributed variables. The chi-square test was used to assess any significant associations between the categorical variables. The independent-samples $t$-test was utilized to compare differences in the mean between the groups (adjusted for age and BMI). The paired-samples $t$-test and the Wilcoxon signed-rank test were applied to compare any differences over time for the normally and non-normally distributed independent continuous variables, respectively. A binary logistic regression model was explored the predictors of mortality during the early stay in hospitals. The Cox regression for survival analysis was also investigated the effect of predictors upon the time of mortality during the early stay in hospitals. A p-value of $<0.050$ was statistically significant.

\section{Results \\ Demographic Characteristics and Comorbidities}

The mortality rate of COVID-19 patients was reported to be 3\% between July 1, 2020, and December 31, 2020, in this study. Table 1 shows the demographic characteristics 


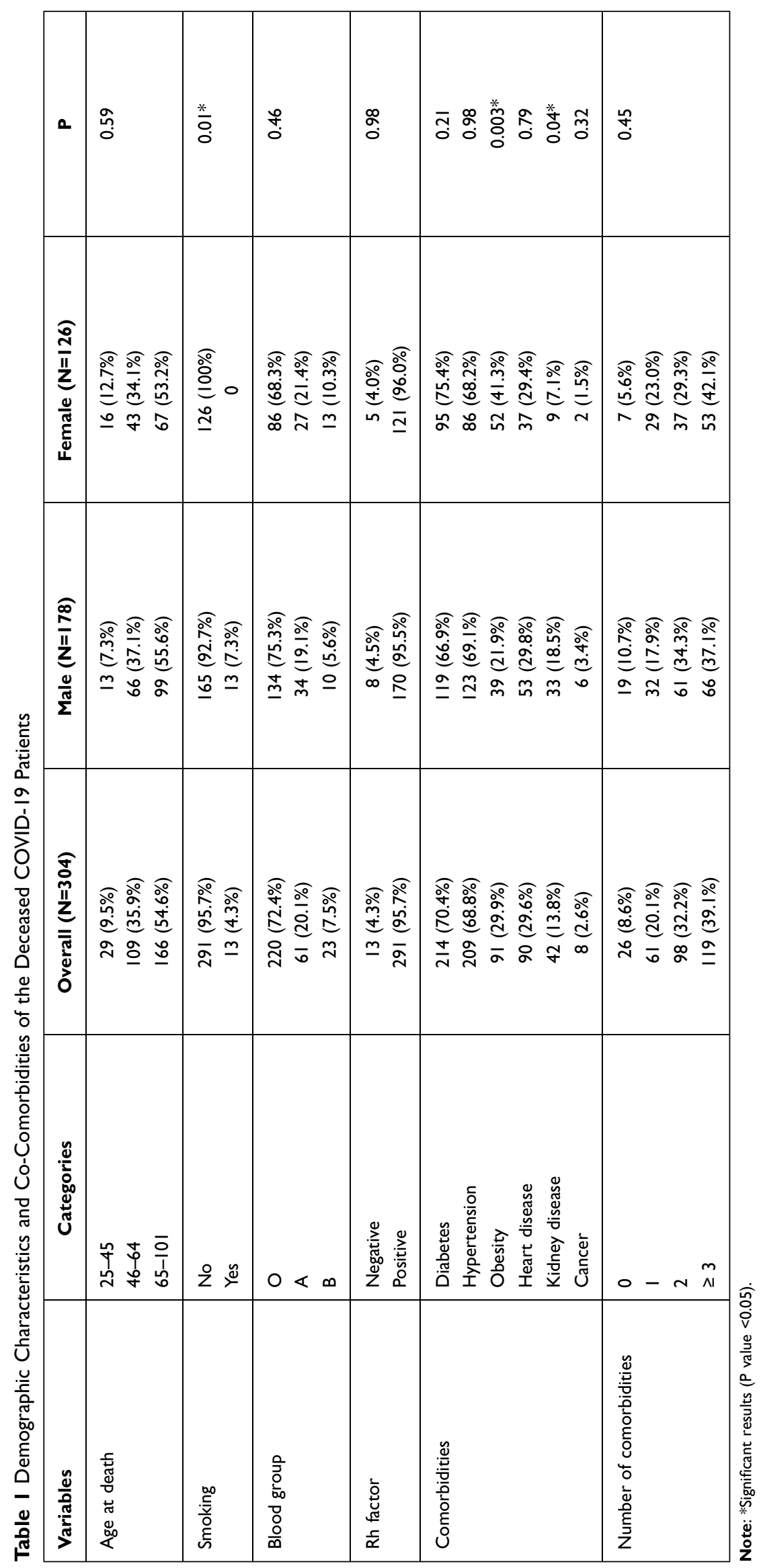


Table 2 Clinical Symptoms and Signs on Admission Adjusted for Age and BMI

\begin{tabular}{|c|c|c|c|c|}
\hline Clinical Symptoms \& Signs & Overall $(\mathrm{N}=304)$ & Male $(\mathrm{N}=\mid 78)$ & Female $(\mathrm{N}=126)$ & $\mathbf{P}$ \\
\hline Dyspnea & $275(90.5 \%)$ & $159(89.3 \%)$ & $116(92.1 \%)$ & 0.46 \\
\hline Cough & $244(80.3 \%)$ & I 44 (80.9\%) & 100 (79.4\%) & 0.81 \\
\hline Desaturation $\left(\mathrm{SpO}_{2}<94 \%\right)$ & $222(73.0 \%)$ & $126(70.8 \%)$ & $96(76.1 \%)$ & 0.47 \\
\hline Fever & $214(70.4 \%)$ & $125(70.2 \%)$ & 89 (70.6\%) & 0.79 \\
\hline Confusion & 40 (I3.2\%) & $26(14.6 \%)$ & 14 (II.1\%) & 0.55 \\
\hline Diarrhea & 39 (I2.8\%) & $23(12.9 \%)$ & $16(12.7 \%)$ & 0.98 \\
\hline Chest pain & 18 (5.9\%) & $10(5.6 \%)$ & $8(6.3 \%)$ & 0.78 \\
\hline Temperature $\left(\mathrm{C}^{\circ}\right)^{*}$ & $37.2 \pm 0.9$ & $37.2 \pm 0.9$ & $37.3 \pm 1.0$ & 0.62 \\
\hline Systolic/diastolic Blood pressure ${ }^{\#}(\mathrm{mmHg})^{*}$ & $122.9 \pm 24.3 / 68.2 \pm 14.3$ & $124.5 \pm 24.7 / 68.6 \pm 14.1$ & $122.4 \pm 24.6 / 66.9 \pm 15.0$ & 0.55 \\
\hline $\mathrm{SpO}_{2}^{\wedge}(\%)^{*}$ & $86.5 \pm 13.3$ & $87.6 \pm 10.6$ & $85.7 \pm 16.1$ & 0.32 \\
\hline
\end{tabular}

Notes: *Independent-samples t-test compared means difference between male and female groups.

and co-comorbidities of the 304 hospitalized COVID-19 patients who died, stratified according to sex. A greater proportion of male patients $(59 \%)$, compared to female patients (41\%), died due to COVID-19 (Table 1). Just over half $(55 \%)$ of deaths due to COVID-19 occurred in patients aged $\geq 65$ years (Table 1 ). The mean age of the patients was 65.9 years \pm 15.6 (range of $25-101$ years). Most male COVID-19 patients who died (96\%) were nonsmokers; however, the number of deceased male smokers was greater than the number of deceased female smokers $(p=0.010)($ Table 1). More than two-thirds of the patients (72\%) who died due to COVID-19 had blood group type O (Table 1). A similar proportion had diabetes $(70 \%)$ and hypertension $(69 \%)$, followed by heart disease $(30 \%)$ and chronic kidney disease (14\%). Mean BMI was $27.4 \mathrm{~kg} / \mathrm{m}^{2}$ \pm 5.8 (range of $13.8-45.2 \mathrm{~kg} / \mathrm{m} 2$ ). Almost one-third of hospitalized COVID-19 patients who demised were obese $(30 \%)$, and a greater number of obese women died, compared to obese men $(p=0.003)($ Table 1$)$.

\section{Clinical Symptoms and Hospitalization Length of Stay}

The most frequently reported clinical symptoms of hospitalized Saudi patients with COVID-19 who died were dyspnea (91\%), cough (80\%), and fever $(70 \%)$; a small percentage reported chest pain (6\%) (Table 2). Differences in the patients' clinical symptoms by gender were not identified. Eighty-five per cent of patients who died due to COVID-19 were admitted to the intensive care unit (ICU) and, of the patients, 90\% required mechanical ventilation. The median duration between diagnosis and death, hospital admission and death, and ICU admission and death, was 12, 9, and 8 days, respectively (Figure 1). Details of the hospitalization duration (in weeks) and data for the prediction of hospital mortality in COVID-19 patients (using the Acute Physiology and Chronic Health Evaluation II [APACHE II]) are provided in Figures 2 and 3. Most COVID-19 patients (66\%) had an APACHE II score of $\geq 30$. Comorbidities were evaluated using the chisquare test, and it was established that a significantly high proportion of COVID-19 patients who died had diabetes and were admitted to ICU $(p=0.001)$.

\section{Clinical Laboratory Findings}

The clinical laboratory findings of hospitalized COVID-19 patients who died, at admission and 24 hours prior to death were analyzed and adjusted for hypertension, heart disease, diabetes, kidney disease, and cancer. The clinical laboratory findings changed in most of the conducted tests (Table 3). Typically, leukocytosis, lymphocytopenia, and neutrophilia were observed upon admission and 24-hr prior to death (Table 3). Patients who demised due to COVID-19 had high levels of ferritin, lactate dehydrogenase, D-dimer, and erythrocyte sedimentation rate at admission and 24 hours prior to death (Table 3).

\section{Radiological and Electrocardiogram Investigations}

The findings of the radiological and electrocardiographic investigations are provided in Table 4. Lung infiltrates and 


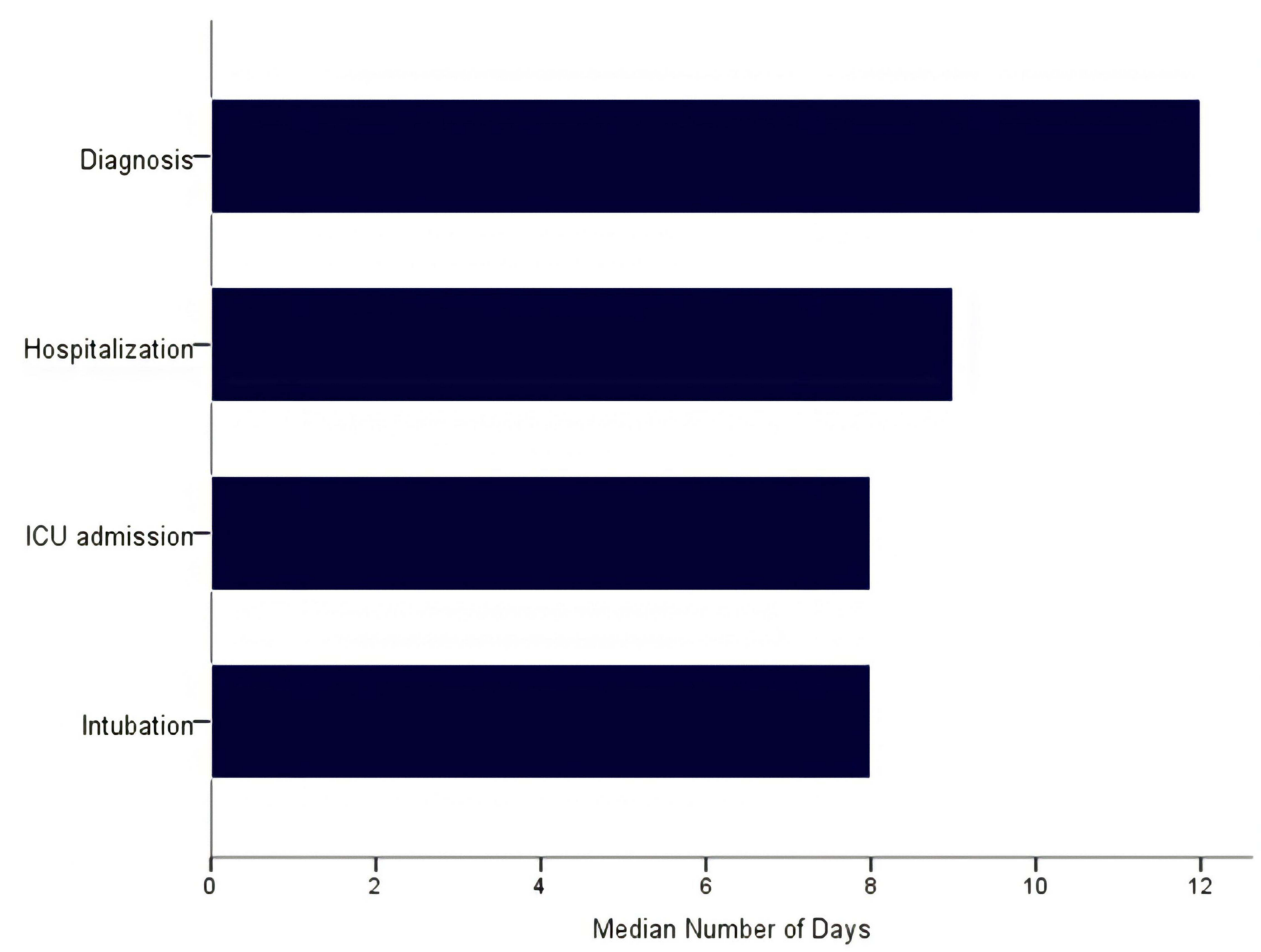

Figure I Median number of days from confirmed COVID-19 diagnosis to (X) event.

pulmonary opacity were identified in the majority of hospitalized COVID-19 patients (83\%). Differences in the radiological findings were not observed by gender.

\section{Associated Complications}

Associated complications were analysed (Table 5). Respiratory failure $(70 \%)$ and acute respiratory distress syndrome (52\%) were the leading causes of death, followed by sepsis (35\%), and acute kidney injury (30\%) (Table 5).

\section{Clinical Management}

Among the supportive medications provided, antiviral agents (favipiravir), immunosuppressive agents (tocilizumab), antibiotics (azithromycin), corticosteroids (methylprednisolone), and anticoagulant agents (enoxaparin) were the most common medications given to the COVD-19 patients in the current study (Table 6).

\section{Logistic Regression and Proportional Hazards Analysis}

Logistic regression and Cox proportional hazards model revealed that a higher age led to increased risk of mortality among COVID-19 patients with a short stay hospitalization.
Such risk was significantly high in patients aged greater than 65 years, who had more than two times the risk of mortality compared to other age groups (Tables 7 and 8). Smoking, high creatinine and aspartate transaminase levels, and respiratory failure were also significantly associated with the risk of mortality during the early stay in hospitals.

\section{Discussion}

COVID-19 is a novel disease and, as such, the current study aimed to address the gap in knowledge regarding the epidemiological and clinical characteristics of Saudi patients with COVID-19 who died in Jazan, Saudi Arabia. The mortality rate of COVID-19 patients was reported to be $3 \%$ between July 1, 2020, and December 31,2020 , in this study, and it was estimated to be $4 \%$ in February 2021 in Jazan Region, Saudi Arabia by the Saudi Ministry of Health, ${ }^{2}$ which indicates that the number of deaths is increasing significantly over time. However, this rate is lower than that reported in Wuhan, China. ${ }^{8,16}$

In the current study, most COVID-19 patients who died were aged $\geq 65$ years $(55 \%)$, and old age was a predictor for early mortality during short stay hospitalization, which is consistent with the findings of other studies. ${ }^{3,8,17}$ The 


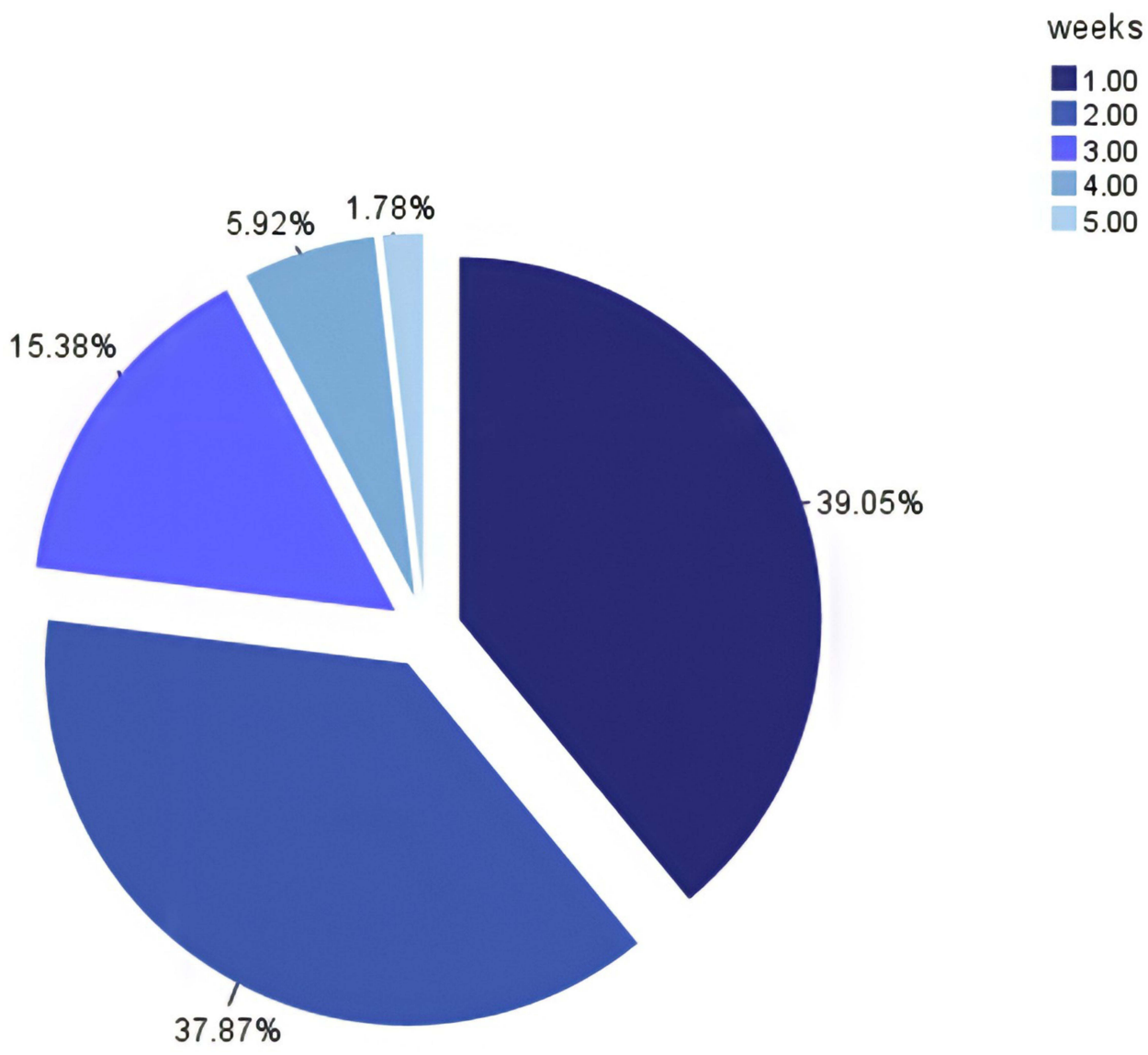

Figure 2 Percentage (\%) of patients who were hospitalized in weeks to death.

immune response decreases with age, which, in turn, increases the risk of death due to COVID-19. ${ }^{18,19}$ Most COVID-19 patients who died belonged to blood group O (72\%); however, an association between blood group and mortality risk related to COVID-19 was not identified in a previous study.$^{20}$ Thus, it is likely that blood group $\mathrm{O}$ is prevalent in the Saudi population living in Jazan Region. Most patients who died of COVID-19 in the current study had two or more comorbidities, with diabetes being the most prevalent (70\%). These results are consistent with the findings of a previous report. ${ }^{8,16}$

Respiratory failure (70\%) and acute respiratory distress syndrome $(52 \%)$ were the most common complications leading to death in COVID-19 patients. A similar finding was reported elsewhere. ${ }^{8}$ Human airway epithelia express angiotensin-converting enzyme 2 (ACE2), a host cell receptor for SARS-CoV-2 infection. Of all organs, the lungs are most impacted by SARS-CoV-2 infection. ${ }^{21}$ In one study, the pathological findings indicated that patients whose lungs were infected with SARS-CoV-2 presented with acute respiratory distress syndrome, pulmonary hyaline membrane formation, and evidence of pneumocyte desquamation. $^{22}$ ACE2 expression also takes place in the heart, liver, and kidney organs, and damage to these organs, due to the invasion of SARS-CoV-2, was observed in the current study using the laboratory findings. ${ }^{8}$

The laboratory parameters were predictive of severe COVID-19 symptoms and the risk of early mortality. In the current study, white blood cell (WBC) count was elevated $\left(>10 \times 10^{9}\right.$ cells/L) upon admission and increased significantly prior to death $\left(15.5 \times 10^{9}\right.$ cells $\left./ \mathrm{L}\right)$. The elevation in WBC count correlated with disease severity. ${ }^{23}$ In addition, an increase in neutrophils (median of $12.9 \times 10^{9}$ cells $/ \mathrm{L}$ ) and a decrease in lymphocytes (median of $0.5 \times 10^{9}$ cells $/ \mathrm{L}$ ) was also observed prior to death. This finding corresponds with that of a previous study that reported that COVID-19 patients with severe symptoms had more neutrophils and fewer lymphocytes compared to COVID-19 patients with 


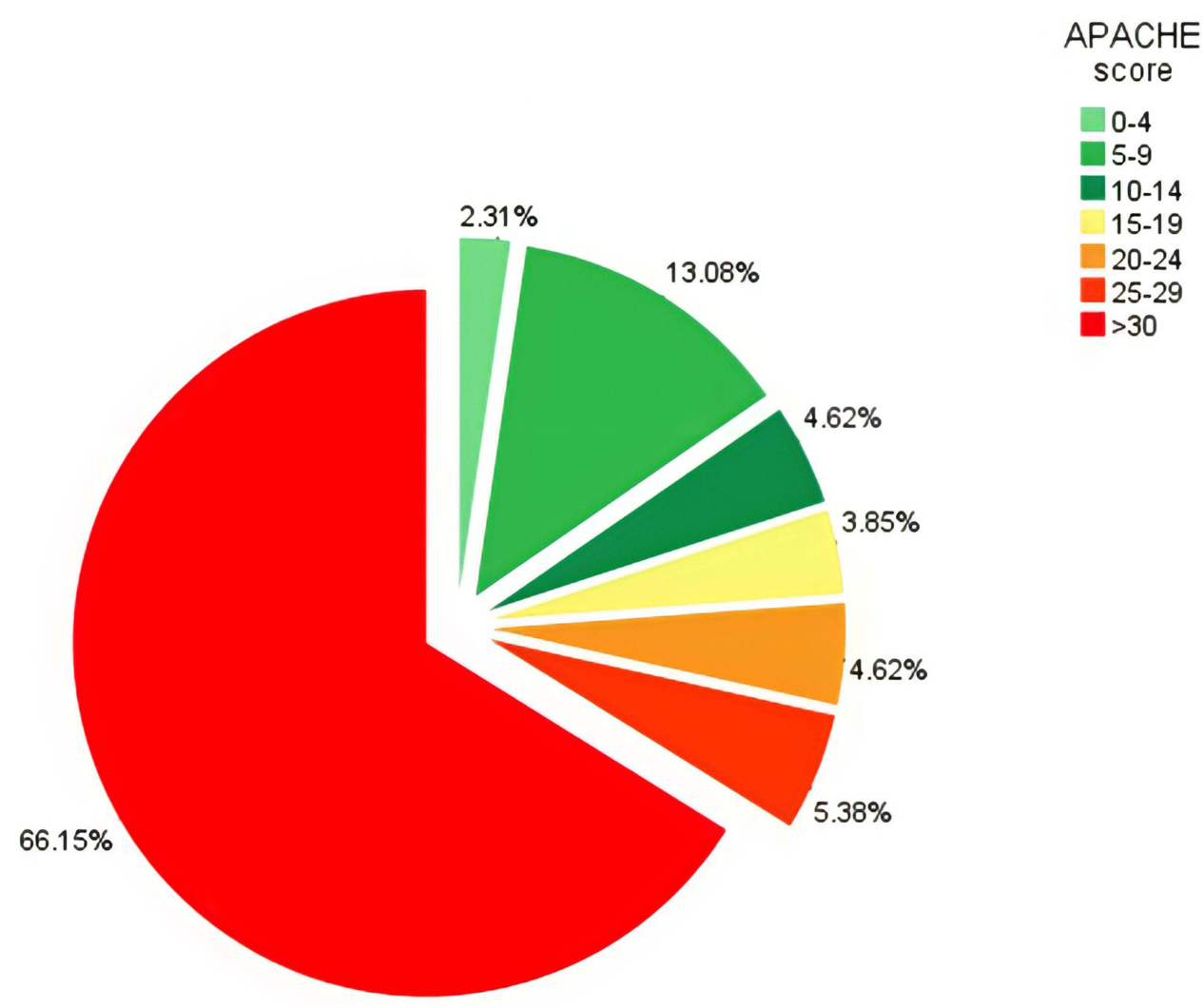

Figure 3 Percentage (\%) of patients with APACHE scores at admission.

non-severe disease. ${ }^{16}$ The age of COVID-19 patients may be used to determine the risk of lymphocytopenia and its progression, ${ }^{24}$ while lymphocytopenia can be utilized to predict disease severity. ${ }^{25,26}$ It has been hypothesized that lymphocytopenia is associated with the inflammatory process (cytokine storm syndrome), direct lymphocyte infection, and lymphoid organ destruction. ${ }^{25,27}$ The administration of glucocorticosteroids in the treatment of COVID-19 patients may lead to lymphocytopenia. ${ }^{28}$

The patient's hemoglobin levels were within the normal range in the current study, similar to the finding of previous research. ${ }^{23,24,29-31}$ However, patients with severe symptoms exhibited lower hemoglobin levels, compared to those with mild symptoms, in one metaanalysis study. ${ }^{32}$ Platelet count was also within the normal range in the current research; similarly, thrombocytopenia was not identified in other similar studies that investigated this. ${ }^{16,23,29-31}$ An increase in serum ferritin levels was observed in COVID-19 patients upon admission and prior to death (median of 774 , IQR of 404-1845). These results mirror those of previous studies. ${ }^{29,30}$ This finding may be elucidated by severe inflammation as ferritin is a positive acute-phase protein. ${ }^{33}$ D-dimer levels were also markedly increased in the COVID-19 patients under investigation, and this finding is supported elsewhere. ${ }^{23,29,30,34}$ An increase in D-dimer levels is associated with high fibrin degradation products levels and low antithrombin activity, as well as the risk of thrombotic and hemorrhagic complications. $^{35,36}$ The rate of erythrocyte sedimentation was also significantly elevated in the current study, and this finding is corroborated in other studies. ${ }^{37,38}$

Most of these risk factors are linked, in varying degrees, to the progression of acute respiratory distress syndrome secondary to SARS-CoV-2, and they contribute to the exacerbation of pre-existing chronic inflammation, which then progresses to cytokine storm and the rapid impairment of endothelial function. ${ }^{39}$ 
Table 3 Clinical Laboratory Findings of Hospitalized COVID-19 Deaths

\begin{tabular}{|c|c|c|c|}
\hline Variables & At Admission & 24-hr Prior to Death & $\mathbf{P}$ \\
\hline \multicolumn{4}{|l|}{ Complete blood count } \\
\hline Hemoglobin ${ }^{\#}$ (mmol/L) & $11.6 \pm 2.3$ & $10.3 \pm 2.3$ & $<0.00 I^{*}$ \\
\hline White blood cell^ (xI09/L) & $10.8(7.1-15.6)$ & $15.5(10.7-23.4)$ & $<0.00 I^{*}$ \\
\hline Platelets ${ }^{\#}(x \mid 09 / L)$ & $251.8 \pm 104.7$ & $174.1 \pm 105.4$ & $<0.00 I^{*}$ \\
\hline Neutrophils^ (\%) & $6.9(4.8-9.1)$ & $12.9(5.4-13.1)$ & $<0.00 I^{*}$ \\
\hline Lymphocytes^ (\%) & $0.7(0.5-0.9)$ & $0.5(0.3-0.8)$ & $0.010 *$ \\
\hline Serum ferritin ${ }^{\wedge}(\mathrm{pmol} / \mathrm{L})$ & $623(328.3-1236)$ & $774(404.5-1845)$ & $0.010^{*}$ \\
\hline \multicolumn{4}{|l|}{ Coagulation profile } \\
\hline D-dimer^ (mcg/mL) & $2.1(0.9-8.2)$ & $5.1(2.5-13)$ & 0.003 \\
\hline \multicolumn{4}{|l|}{ Electrolytes } \\
\hline Sodium $\#$ (mmol/L) & $137.9 \pm 8.3$ & $142.2 \pm 8.0$ & $<0.00 I^{*}$ \\
\hline Calcium^ (mmol/L) & $2.1(1.9-2.2)$ & $2.0(1.8-2.2)$ & 0.057 \\
\hline Potassium ${ }^{\#}$ (mmol/L) & $4.5 \pm 0.9$ & $4.9 \pm 1.1$ & $<0.00 I^{*}$ \\
\hline \multicolumn{4}{|l|}{ Enzymes } \\
\hline Lactate dehydrogenase (unit/L) & $511(307-676)$ & $67 \mid(472-1164)$ & $<0.00 I^{*}$ \\
\hline Creatine kinase ${ }^{\wedge}(\mu \mathrm{mol} / \mathrm{L})$ & $120(51.3-444.6)$ & I 35 (7|.4-455.8) & 0.102 \\
\hline \multicolumn{4}{|l|}{ Renal profile } \\
\hline Creatinine $^{\wedge}(\mu \mathrm{mol} / \mathrm{L})$ & $105.8(77.9-185.5)$ & $165(80.5-400)$ & $<0.00 I^{*}$ \\
\hline Blood urea nitrogen^ (mmol/L) & $13(6.4-23.9)$ & $22.2(11.8-40.8)$ & $<0.001 *$ \\
\hline \multicolumn{4}{|l|}{ Liver profile } \\
\hline Alanine aminotransferase (unit/L) & $33(20.3-8 \mid .2)$ & $49.5(31.0-85.2)$ & $0.001 *$ \\
\hline Aspartate transaminase (unit/L) & $50.2(31.0-86.8)$ & $56.8(33-123.5)$ & $0.006 *$ \\
\hline Bilirubin^ (umol/L) & $9.5(5.0-18.7)$ & $16.2(8.7-34.3)$ & $<0.00 I^{*}$ \\
\hline Albumin $^{\#}(g / L)$ & $26.8 \pm 9.1$ & $24.1 \pm 8.4$ & $<0.00 I^{*}$ \\
\hline \multicolumn{4}{|l|}{ Inflammatory profile } \\
\hline Erythrocyte sedimentation rate $(\mathrm{mm} / \mathrm{hr})$ & $85(62.8-117.5)$ & $80(59.8-95.3)$ & 0.820 \\
\hline Glucose Random ${ }^{\#}$ (mmol/L) & $9.6 \pm 4.9$ & $11.7 \pm 6.8$ & 0.069 \\
\hline
\end{tabular}

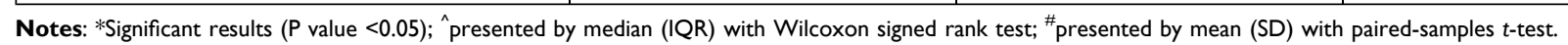

Table 4 Findings of Radiological and Electrocardiogram Investigations

\begin{tabular}{|c|c|c|c|c|c|}
\hline Investigation & Findings & Overall $(N=304)$ & Male $(N=\mid 78)$ & Female $(\mathrm{N}=126)$ & $\mathbf{P}$ \\
\hline Chest X-Ray & $\begin{array}{l}\text { Normal } \\
\text { Bilateral } \\
\text { Infiltrate/opacities } \\
\text { Pleural effusion }\end{array}$ & $\begin{aligned} 7 & (2.3 \%) \\
43 & (14.1 \%) \\
251 & (82.5 \%) \\
& 3(1.1)\end{aligned}$ & $\begin{array}{c}7(3.9 \%) \\
21(11.8 \%) \\
148(83.1 \%) \\
2(1.2 \%)\end{array}$ & $\begin{array}{c}0 \\
22(17.5 \%) \\
103(81.7 \%) \\
1(0.8 \%)\end{array}$ & 0.29 \\
\hline ECG* & $\begin{array}{l}\text { Normal } \\
\text { Non-ST elevation } \\
\text { QTc prolongation }\end{array}$ & $\begin{array}{c}292(96.1 \%) \\
8(2.6 \%) \\
4(1.3 \%)\end{array}$ & $\begin{array}{c}166(93.4 \%) \\
8(4.4 \%) \\
4(2.2 \%)\end{array}$ & $\begin{array}{c}126(100 \%) \\
0 \\
0\end{array}$ & 0.15 \\
\hline
\end{tabular}

Note: *Electrocardiogram. 
Table 5 Complications Associated with Cause of Death

\begin{tabular}{|l|c|c|c|c|}
\hline Complications & Overall (N=304) & Male (N=I 78) & Female (N=126) & P \\
\hline Any (No complications) & $13(4.2 \%)$ & $9(5.1 \%)$ & $89(70.6 \%)$ & 0.24 \\
\hline Respiratory failure & $212(69.7 \%)$ & $123(69.1 \%)$ & $72(57.1 \%)$ & 0.83 \\
\hline Acute respiratory distress syndrome & $159(52.3 \%)$ & $87(48.9 \%)$ & $48(38.1 \%)$ & 0.32 \\
\hline Sepsis/septic shock & $106(34.9 \%)$ & $58(32.6 \%)$ & $40(31.7 \%)$ & 0.42 \\
\hline Acute kidney injury & $93(30.6 \%)$ & $53(29.8 \%)$ & $6(4.8 \%)$ & 0.76 \\
\hline Secondary infections & $19(6.3 \%)$ & $13(7.3 \%)$ & $3(2.4 \%)$ & 0.55 \\
\hline Heart failure & $16(5.3 \%)$ & $13(7.3 \%)$ & $3(2.4 \%)$ & 0.16 \\
\hline Acute pulmonary embolism & $11(3.6 \%)$ & $8(4.5 \%)$ & $5(3.9 \%)$ & 0.48 \\
\hline Cardiogenic shock & $10(3.3 \%)$ & $5(2.8 \%)$ & $3(2.4 \%)$ & 0.67 \\
\hline Ischemic stroke & $8(2.6 \%)$ & $5(2.8 \%)$ & $1(1.3 \%)$ & 0.95 \\
\hline Atrial fibrillation & $5(1.6 \%)$ & $5(2.8 \%)$ & 0 & 0.49 \\
\hline Myocardial infarction & $6.8 \%)$ & & $0.03 *$ \\
\hline
\end{tabular}

Note: *Significant results $(\mathrm{P}$ value $<0.05)$.

Table 6 Medications That Were Given to Hospitalized COVID-19 Deaths

\begin{tabular}{|l|c|c|c|c|}
\hline Medications & Overall (N=304) & Male (N=I78) & Female (N=126) & P \\
\hline Favipiravir & $224(73.8 \%)$ & $122(68.5 \%)$ & $13(10.3 \%)$ & 0.07 \\
\hline Lopinavir/Ritonavir & $32(10.5 \%)$ & $19(10.7 \%)$ & $13(10.3 \%)$ & 0.89 \\
\hline Ribavirin & $34(11.2 \%)$ & $21(11.8 \%)$ & $3(2.4 \%)$ & 0.74 \\
\hline Remdesivir & $6(2.0 \%)$ & $3(1.7 \%)$ & $2(1.6 \%)$ & 0.73 \\
\hline Hydroxychloroquine & $5(1.6 \%)$ & $3(1.7 \%)$ & $64(50.8 \%)$ & 0.77 \\
\hline Tocilizumab & $124(40.8 \%)$ & $60(33.7 \%)$ & $55(43.6 \%)$ & $0.01 *$ \\
\hline Azithromycin & $136(44.7 \%)$ & $81(45.5 \%)$ & $57(45.2 \%)$ & 0.80 \\
\hline Methylprednisolone & $141(46.3 \%)$ & $84(47.2 \%)$ & $97(76.9 \%)$ & 0.75 \\
\hline Enoxaparin & $234(77.0 \%)$ & $137(76.9 \%)$ & $32(25.4 \%)$ & 0.95 \\
\hline Heparin & $87(28.6 \%)$ & $55(30.9 \%)$ & $9(7.1 \%)$ & 0.43 \\
\hline ACEl/ARBS & $22(7.2 \%)$ & $13(7.3 \%)$ & $111(88.1 \%)$ & 0.91 \\
\hline DVT* prophylaxis & $276(90.8 \%)$ & $165(92.7 \%)$ & $58(46.0 \%)$ & 0.45 \\
\hline DVT* treatment & $127(41.8 \%)$ & $69(38.8 \%)$ & 0.41 \\
\hline Not & $13 \%$ & \\
\hline
\end{tabular}

Notes: *Significant results (P value $<0.05$ ); \#angiotensin-converting enzyme inhibitors/ angiotensin II receptor blockers; *deep vein thrombosis.

\section{Conclusion}

The proportion of comorbidities was high in deceased patients who were hospitalized with COVID-19 in Jazan region, Saudi Arabia. A higher age, smoking, and respiratory failure were significant predictors of mortality during the early stay in hospitals. 
Table 7 Predictors of Mortality in COVID-19 Patients During the Early Stay in Hospitals Using Multivariate Logistic Regression

\begin{tabular}{|l|c|c|c|}
\hline \multirow{2}{*}{ Predictors } & \multicolumn{3}{|c|}{ Logistic Regression } \\
\cline { 2 - 4 } & OR & $95 \%$ CI & p-value \\
\hline Smoking & 4.0 & $1.4-5.8$ & $0.003^{*}$ \\
\hline Respiratory failure & 2.4 & $0.2-3.1$ & $0.02^{*}$ \\
\hline Age > 65 years & 2.1 & $1.6-2.8$ & $0.005^{*}$ \\
\hline Myocardial infarction & 2.1 & $0.3-3.9$ & 0.57 \\
\hline Aspartate transaminase & 1.9 & $0.9-3.6$ & $0.02 *$ \\
\hline Creatinine & 1.4 & $0.9-1.9$ & $0.02 *$ \\
\hline Diabetes & 1.4 & $0.7-3.1$ & 0.37 \\
\hline Sex & 1.2 & $0.6-2.4$ & 0.65 \\
\hline Cardiovascular disease & 1.2 & $0.6-2.7$ & 0.59 \\
\hline Sepsis/septic shock & 1.2 & $0.6-2.5$ & 0.57 \\
\hline Chronic kidney disease & 1.1 & $0.2-1.4$ & 0.19 \\
\hline Alanine aminotransferase & 1.1 & $0.4-1.3$ & 0.68 \\
\hline Pulmonary embolism & 1.1 & $0.1-3.1$ & 0.87 \\
\hline D-dimer & 1.0 & $0.9-1.1$ & 0.33 \\
\hline Obesity & 1.0 & $0.9-1.2$ & 0.37 \\
\hline Hypertension & 1.0 & $0.4-2.0$ & 0.84 \\
\hline Note: & & & \\
\hline
\end{tabular}

Note: *Significant results $(\mathrm{P}$ value $<0.05)$.

\section{Data Sharing Statement}

The data that support the findings of this study are available on request from the corresponding author, Mohammed Badedi.

\section{Ethics Approval}

The Jazan Health Ethics Committee (Reference number: H-10-Z-073) granted ethical approval (No. 2030) for the study to be conducted, and the research complied with the Declaration of Helsinki. Consent was waived as data were anonymous and collected retrospectively (secondary data).

\section{Disclosure}

The authors report no conflict of interests in this work.

\section{References}

1. World Health Organization (WHO). Coronavirus Disease (COVID19): Weekly Epidemiological Update. Geneva: WHO; 2020.

2. Ministry of Health (MOH). Coronavirus Disease (COVID-19): Daily Report Update. Riyadh: MOH; 2020.
Table 8 Predictors of Mortality in COVID-19 Patients During the Early Stay in Hospitals Using Cox Regression

\begin{tabular}{|l|c|c|c|}
\hline \multirow{2}{*}{ Predictors } & \multicolumn{3}{|c|}{ Cox Regression } \\
\cline { 2 - 4 } & HR & $95 \%$ CI & p-value \\
\hline Smoking & 3.0 & $1.2-4.3$ & $0.008^{*}$ \\
\hline Respiratory failure & 2.7 & $0.9-3.2$ & $0.01^{*}$ \\
\hline Age > 65 years & 2.4 & $1.8-2.9$ & $0.001^{*}$ \\
\hline Aspartate transaminase & 2.3 & $1.1-4.3$ & $0.01^{*}$ \\
\hline Creatinine & 1.9 & $0.9-2.3$ & $0.02^{*}$ \\
\hline Myocardial infarction & 1.7 & $0.4-3.8$ & 0.50 \\
\hline Diabetes & 1.5 & $1.1-2.6$ & 0.14 \\
\hline Sepsis/septic shock & 1.4 & $0.4-1.2$ & 0.23 \\
\hline Chronic kidney disease & 1.3 & $0.6-2.9$ & 0.45 \\
\hline Alanine & 1.3 & $0.7-1.8$ & 0.89 \\
\hline aminotransferase & & & 0.7 \\
\hline Obesity & 1.2 & $0.7-2.1$ & 0.48 \\
\hline Cardiovascular disease & 1.2 & $0.7-2.1$ & 0.51 \\
\hline Sex & 1.1 & $0.6-1.9$ & 0.75 \\
\hline Hypertension & 1.1 & $0.5-1.7$ & 0.88 \\
\hline D-dimer & 1.0 & $0.9-1.3$ & 0.18 \\
\hline Pulmonary embolism & 1.0 & $0.3-2.9$ & 0.98 \\
\hline Note: Sig & \\
\hline
\end{tabular}

Note: *Significant results $(\mathrm{P}$ value $<0.05)$.

3. Badedi M, Makrami A, Alnami A. Co-morbidity and blood group type risk in coronavirus disease 2019 patients: a case-control study. $J$ Infect Public Health. 2021;14(4):550-554. doi:10.1016/j.jiph.2020.12.035

4. Bai Y, Yao L, Wei T, et al. Presumed asymptomatic carrier transmission of COVID-19. JAMA. 2020;323(14):1406-1407. doi:10.1001/ jama.2020.2565

5. Ye F, Xu S, Rong Z, et al. Delivery of infection from asymptomatic carriers of COVID-19 in a familial cluster. Int $J$ Infect Dis. 2020;94:133-138. doi:10.1016/j.ijid.2020.03.042

6. Verity R, Okell L, Dorigatti I, et al. Estimates of the severity of coronavirus disease 2019: a model-based analysis. Lancet Infect Dis. 2020;20:669-677. doi:10.1016/S1473-3099(20)30243-7

7. Yuki K, Fujiogi M, Koutsogiannaki S. COVID-19 pathophysiology: a review. Clin Immunol. 2020;215:108427. doi:10.1016/j. clim.2020.108427

8. Zhang B, Zhou X, Qiu Y, et al. Clinical characteristics of 82 cases of death from COVID-19. PLoS One. 2020;15(7):e235458. doi:10.1371/journal.pone. 0235458

9. Panthee B, Dhungana S, Panthee N, Gyawali S, Paudel A, Panthee S. Clinical and epidemiological features of COVID-19 deaths in Nepal. New Microbes New Infect. 2020;38:100797. doi:10.1016/j.nmni.2020.100797

10. Badedi M, Darraj H, Hummadi A, et al. Khat chewing and type 2 diabetes mellitus. Diabetes Metab Syndr Obes. 2020;13:307-312.

11. Alzughbi T, Badedi M, Darraj H, et al. Diabetes-related distress and depression in Saudis with type 2 diabetes. Psychol Res Behav Manag. 2020;13:453-458. doi:10.2147/PRBM.S255631 
12. Badedi M, Darraj H, Hummadi A, et al. Vitamin B12 deficiency and foot ulcers in type 2 diabetes mellitus: a Case-Control Study. Diabetes Metab Syndr Obes. 2019;12:2589-2596. doi:10.2147/ DMSO.S233683

13. Rees E, Nightingale E, Jafari Y, et al. COVID-19 length of hospital stay: a systematic review and data synthesis. BMC Med. 2020;18 (1):270. doi:10.1186/s12916-020-01726-3

14. World Health Organization (WHO). Physical status: the use and interpretation of anthropometry: report of a WHO expert committee. Technical report series 854. Geneva: WHO; 1995.

15. BM Corp. IBM SPSS Statistics for Windows. NY: IBM Corp; 2012.

16. Wang D, Hu B, Hu C, et al. Clinical characteristics of 138 hospitalized patients with 2019 novel coronavirus-infected pneumonia in Wuhan, China. JAMA. 2020;323(11):1061-1069. doi:10.1001/jama.2020.1585

17. Zhou F, Yu T, Du R, et al. Clinical course and risk factors for mortality of adult inpatients with COVID-19 in Wuhan, China: a retrospective cohort study. Lancet. 2020;395:1054-1062. doi:10.1016/S0140-6736(20)30566-3

18. Goronzy J, Fang F, Cavanagh M, Qi Q, Weyand C. Naive T cell maintenance and function in human aging. J Immunol. 2015;194 (9):4073-4080. doi:10.4049/jimmunol.1500046

19. Opal S, Girard T, Ely E. The immunopathogenesis of sepsis in elderly patients. Clin Infect Dis. 2005;41(Supplement 7):504-512. doi:10.1086/432007

20. Wu B, Gu D, Yu J, Yang J, Shen W. Association between ABO blood groups and COVID-19 infection, severity and demise: a systematic review and meta-analysis. Infect Genet Evol. 2020;84:104485. doi:10.1016/j.meegid.2020.104485

21. Li W, Moore M, Vasilieva N, et al. Angiotensin-converting enzyme 2 is a functional receptor for the SARS coronavirus. Nature. 2003;426 (6965):450-454. doi:10.1038/nature02145

22. $\mathrm{Xu} \mathrm{Z}$, Shi L, Wang Y, et al. Pathological findings of COVID-19 associated with acute respiratory distress syndrome. Lancet Respir Med. 2020;8(4):420-422. doi:10.1016/S2213-2600(20)30076-X

23. Huang C, Wang Y, Li X, et al. Clinical features of patients infected with 2019 novel coronavirus in Wuhan, China. Lancet. 2020;395 (10223):497-506. doi:10.1016/S0140-6736(20)30183-5

24. Xu X, Wu X, Jiang X, et al. Clinical findings in a group of patients infected with the 2019 novel coronavirus (SARS-Cov-2) outside of Wuhan, China: retrospective case series. BMJ. 2020;368:m606. doi:10.1136/bmj.m606

25. Tan L, Wang Q, Zhang D, et al. Lymphopenia predicts disease severity of COVID-19: a Descriptive and Predictive Study. Signal Transduct Target Ther. 2020;5:33. doi:10.1038/s41392-020-0148-4

26. Li H, Xiang X, Ren H, et al. Serum amyloid A is a biomarker of severe coronavirus disease and poor prognosis. $J$ Infect. 2020;80:646-655. doi:10.1016/j.jinf.2020.03.035
27. Lin L, Lu L, Cao W, Li T. Hypothesis for potential pathogenesis of SARS-CoV-2 infection-a review of immune changes in patients with viral pneumonia. Emerg Microbes Infect. 2020;9:727-732. doi:10.1080/22221751.2020.1746199

28. Yao Z, DuBois D, Almon R, Jusko W. Pharmacokinetic/pharmacodynamic modeling of corticosterone suppression and lymphocytopenia by methylprednisolone in rats. J Pharm Sci. 2008;97:2820-2832. doi:10.1002/jps.21167

29. Chen G, Wu D, Guo W, et al. Clinical and immunological features of severe and moderate coronavirus disease 2019. J Clin Investig. 2020;130:2620-2629. doi:10.1172/JCI137244

30. Liu J, Li S, Liu J, et al. Longitudinal characteristics of lymphocyte responses and cytokine profiles in the peripheral blood of SARSCoV-2 infected patients. EBioMedicine. 2020;55:102763. doi:10.1016/j.ebiom.2020.102763

31. Liu J, Liu Y, Xiang P, et al. Neutrophil-to-lymphocyte ratio predicts critical illness patients with 2019 coronavirus disease in the early stage. J Transl Med. 2020;18:206. doi:10.1186/s12967-020-02374-0

32. Lippi G, Mattiuzzi C. Hemoglobin value may be decreased in patients with severe coronavirus disease 2019. Hematol Transfus Cell Ther. 2020;42:116-117. doi:10.1016/j.htct.2020.03.001

33. Northrop-Clewes C. Interpreting indicators of iron status during an acute phase response-lessons from malaria and human immunodeficiency virus. Ann Clin Biochem. 2008;45:18-32. doi:10.1258/ acb.2007.007167

34. Lippi G, Favaloro E. D-dimer is associated with severity of coronavirus disease 2019: a pooled analysis. Thromb Haemost. 2020;120:876-878. doi:10.1055/s-0040-1709650

35. Han H, Yang L, Liu R, et al. Prominent changes in blood coagulation of patients with SARS-CoV-2 infection. Clin Chem Lab Med. 2020;58:1116-1120. doi:10.1515/cclm-2020-0188

36. Tang N, Li D, Wang X, Sun Z. Abnormal coagulation parameters are associated with poor prognosis in patients with novel coronavirus pneumonia. J Thromb Haemost. 2020;18:844-847. doi:10.1111/jth.14768

37. Wang Z, Yang B, Li Q, Wen L, Zhang R. Clinical features of 69 cases with coronavirus disease 2019 in Wuhan, China. Clin Infect Dis. 2020. doi: $10.1093 / \mathrm{cid} / \mathrm{ciaa} 538$

38. Cai Q, Huang D, Ou P, et al. COVID-19 in a designated infectious diseases hospital outside Hubei Province, China. Allergy. 2020;75 (7):1742-1752. doi:10.1111/all.14309

39. Longo M, Caruso P, Maiorino M, Bellastella G, Giugliano D, Esposito K. Treating type 2 diabetes in COVID-19 patients: the potential benefits of injective therapies. Cardiovasc Diabetol. 2020;19(1):115. doi:10.1186/s12933-020-01090-9
International Journal of General Medicine

\section{Publish your work in this journal}

The International Journal of General Medicine is an international, peer-reviewed open-access journal that focuses on general and internal medicine, pathogenesis, epidemiology, diagnosis, monitoring and treatment protocols. The journal is characterized by the rapid reporting of reviews, original research and clinical studies across all disease areas. The manuscript management system is completely online and includes a very quick and fair peer-review system, which is all easy to use. Visit http://www.dovepress.com/ testimonials.php to read real quotes from published authors. 